\title{
Recording of high efficiency diffraction gratings by He-Ne laser
}

\author{
V.I. Min'ko, P.E. Shepeliavyi, V.A. Dan'ko, P.F. Romanenko, O.S. Litvin, I.Z. Indutnyy \\ V. Lashkaryov Institute of Semiconductor Physics, NAS of Ukraine, 41, prospect Nauky, 03028 Kyiv, Ukraine
}

\begin{abstract}
The investigation results of holographic diffraction gratings recording processes by radiation of helium-neon laser have been represented. Inorganic $\mathrm{As}_{40} \mathrm{~S}_{20} \mathrm{Se}_{40}$ photoresist treated by the newly developed selective etching solution was chosen as a registering media. Angular and spectral dependencies of grating diffractive efficiency absolute values were measured. A comparison of fabricated gratings characteristics with corresponding parameters of gratings recorded on $\mathrm{As}_{40} \mathrm{Se}_{60}$ photoresist was carried out. Numericall simulation of groove profiles inherent to holographic gratings made in various experimental conditions was performed. Shown is that photoresists based on $\mathrm{As}_{40} \mathrm{~S}_{20} \mathrm{Se}_{40}$ are suitable for production of high quality holographic optical elements.
\end{abstract}

Keywords: inorganic photoresist, holographic difraction grating, diffraction efficiency, etching solution.

Paper received 14.02.04; accepted for publication 30.03.04.

\section{Introduction}

Chalcogenide vitreous semiconductors (ChVS) owing to manifold properties have already found various applications, in particular, as inorganic photoresist [1-3]. Photostimulated changes of ChVS layer solubility and optical characteristics serve as a base of their use for manufacturing diffractive optical elements. Holographic diffraction gratings are the simplest among them. Holographic difractive gratings fabrication using inorganic photoresists comprises two main stages: exposure (recording) and after-exposure processing - etching in selective solutions. The quality of produced gratings depends on optimization of these two stages. The first process is determined by the time of exposure, photoresist sensitivity, recording wavelength. The second - by the dissolution rate difference of unexposed and exposed areas of the resist, exposure pattern, time of etching.

Since the spectral light sensitivity distribution of ChVS layers is determined by the absorbed light energy and correlates with the layer absorption spectra [4], then for recording the diffractive optical elements, usually used is radiation of a rather powerful argon laser. Its radiation wavelengths ( 488.0 and $514.5 \mathrm{~nm})$ are in the range of strong absorbance of ChVS photoresists [5]. But, the author of work [6] estimating the photosensitivity range of $\mathrm{As}_{40} \mathrm{~S}_{60-\mathrm{x}} \mathrm{Se}_{\mathrm{x}}$ layers demonstrated possibility to record gratings by He-Ne laser radiation. The process of production of reflective holographic diffraction gratings using He-Ne laser radiation was studied in [7]. It is necessary to note that photosensitivity value at the given actinic wavelength $[3,6]$ depends on both photoresist properties and characteristics of developer used in etching.

In this paper, we report the investigation results of holographic diffraction gratings recording processes using radiation of the most reliable helium-neon laser. Rather frequently used in holography helium-neon laser $(\lambda=632.8 \mathrm{~nm})$ is characterized by stability of generation, absence of water-cooling and operational convenience. Inorganic $\mathrm{As}_{40} \mathrm{~S}_{20} \mathrm{Se}_{40}$ photoresist (vacuum thermally evaporated films) processed by the newly developed selective etching solution was choosen as a registering media. Moreover, a comparison of fabricated gratings characteristics with corresponding parameters of gratings recorded on $\mathrm{As}_{40} \mathrm{Se}_{60}$ photoresist was carried out.

\section{Experiment}

The samples for holographic diffractive grating recording were deposited on high-quality polished glass substrates using thermal vacuum evaporation at the pressure $10^{-5}$ Torr. To provide the necessary adhesion to the 


\section{V.I. Min'ko et al.: Recording of high efficiency diffraction gratings by He-Ne laser}

qlass substrates (it is extremely important for etching process) and to eliminate the interference effects connected with reflectance from the substrate back surface, a chromium layer was deposited on the glass before photoresist deposition. The layer thickness was controled during deposition process by the quartz-crystal-oscillator monitoring system (KIT-1) and reached $60 \mathrm{~nm}$ for chromium layer and $600 \mathrm{~nm}$ for photoresist $\left(\mathrm{As}_{40} \mathrm{~S}_{20} \mathrm{Se}_{40}\right.$ or $\mathrm{As}_{40} \mathrm{Se}_{60}$ ) layer.

Samples for optical measurements were deposited onto polished silica substrates. The transmittance, air-incident, and substrate-incident reflectances of the samples were measured at normal incidence using a spectrophotometer (KSVU-23). The optical constants of the investigated films were calculated, taking into account interference effects, by using these measured values, the sample thickness and the optical constants of the silica substrate (for details, see [8]).

In the course of grating recording, an interference pattern with the spatial frequency of $1130 \mathrm{~mm}^{-1}$ was formed using radiation of the helium-neon laser LHN222. Both photoresists were exposed simultaneously using the same light field intensity distribution. The expose values varied from 0.34 to $2.0 \mathrm{~J} / \mathrm{cm}^{2}$.

After exposure, the samples were chemically treated in non-water alkaline organic solutins. The duration of etching process was chosen equal to the time of complete solution of unexposed photoresist (negative etching). The fabricated relief-phase gratings were covered with reflective thin-film $(\sim 100 \mathrm{~nm})$ Al layer.

Angular (at the wavelength $632.8 \mathrm{~nm}) \eta(\varphi)(\varphi$ is the angle between incidend beams and the normal to the substrate plane) and spectral $\eta(\lambda)(\lambda$-wavelength) dependencies of the diffraction efficiency $\eta$ for the first order of diffraction were measured for $s$ - and $p$-polarizations. The $\eta(\lambda)$ measurements were carried out in $400-800 \mathrm{~nm}$ spectral range in the setup close to the Littrow scheme and the angle between incident and diffracted beams was $4 \mathrm{deg}$. The grating diffraction efficiency was taken as the ratio of intensity of light diffracted into the first order to the intensity of incident light (absolute value of diffractive efficiency). The surface patterns of the gratings were examined with a Dimension 3000 scanning probe microscope (Digital Instruments) in the AFM tapping mode.

\section{Results and discussions}

The exposure dependencies of diffraction efficiency of gratings recorded on $\mathrm{As}_{40} \mathrm{Se}_{60}$ (curve 1) and $\mathrm{As}_{40} \mathrm{~S}_{20} \mathrm{Se}_{40}$ layers (curve 2) are presented in Fig.1 ( $\eta$ was measured at $\lambda=632.8 \mathrm{~nm}$, p-polarization). These dependencies are curves with maxima, which correspond to the optimum exposure $\left(1.34 \mathrm{~J} / \mathrm{cm}^{2}\right)$. The optimum exposure results in the greatest value of diffraction efficiency of gratings. A deviation from this exposure to any direction leads to decrease of diffraction efficiency. Hereafter, all the investigated gratings were recorded at the optimum exposure.

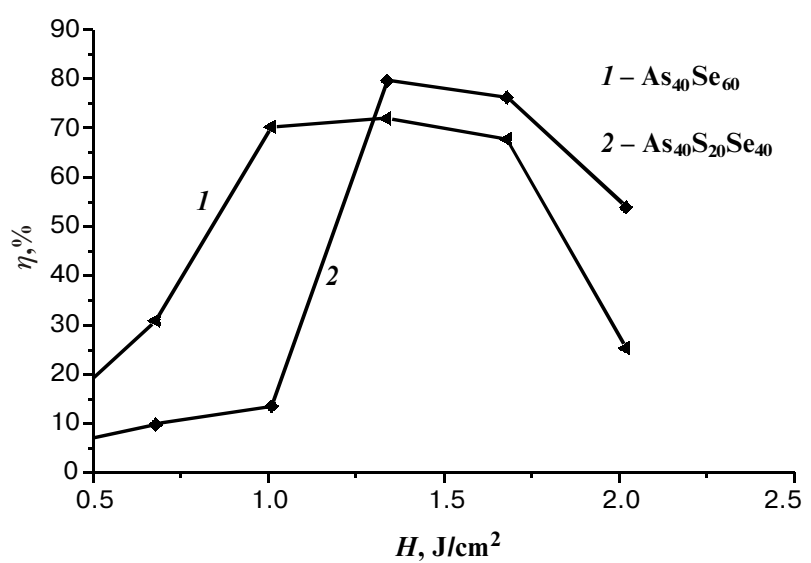

Fig. 1. Expose dependencies of the diffraction efficiency of the gratings recorded using inorganic photoresists.

Fig. 2 shows groove profiles of the gratings obtained using these inorganic photoresists. The groove profiles of both gratings are close to sinusoid, as seen from Fig. 2. The relief depth $\mathrm{h}$ was as large as $316 \mathrm{~nm}$ and $286 \mathrm{~nm}$, the modulation depth ( $h / d$, where $\mathrm{d}$ is grating period) reached 0.36 and 0.32 for gratings on $\mathrm{As}_{40} \mathrm{Se}_{60}$ and $\mathrm{As}_{40} \mathrm{~S}_{20} \mathrm{Se}_{40}$, respectively. Thus, in accordance with [9], we obtained high-modulation gratings. It is necessary to note that absorbance of investigated by us photoresists at the recording wavelength differs nearly four times $\left(1.10^{4} \mathrm{~cm}^{-1}\right.$ $\left.\mathrm{As}_{40} \mathrm{Se}_{60}, 2.7 \cdot 10^{3} \mathrm{~cm}^{-1}-\mathrm{As}_{40} \mathrm{~S}_{20} \mathrm{Se}_{40}\right)$. At the same time,
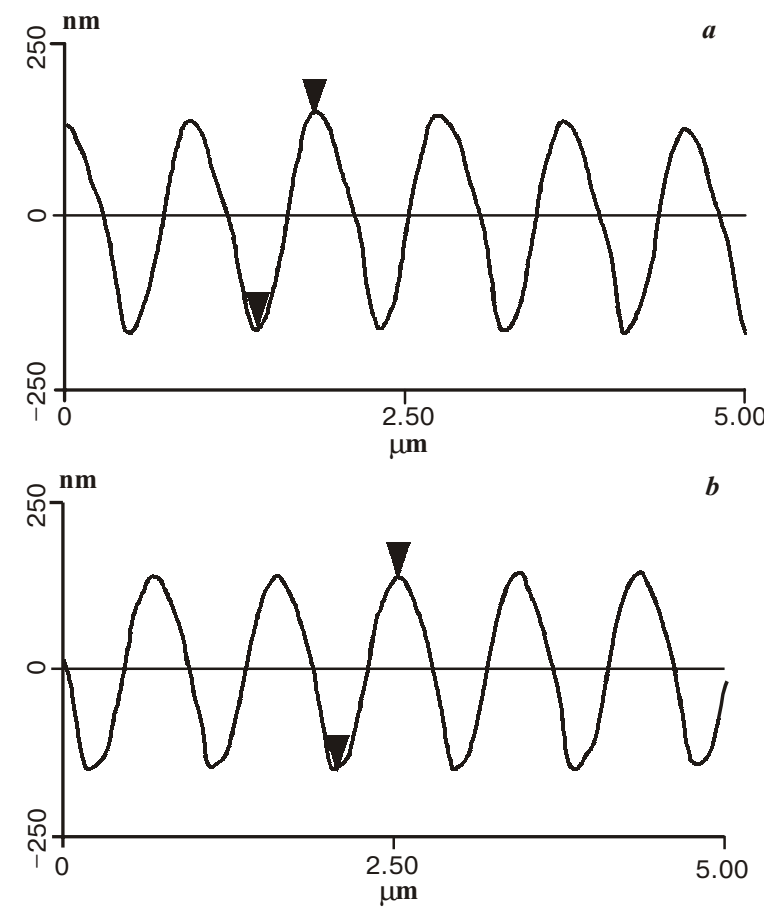

Fig. 2. Relief profiles for the gratings recorded using $\mathrm{As}_{40} \mathrm{Se}_{60}$ (a) and $\mathrm{As}_{40} \mathrm{~S}_{20} \mathrm{Se}_{40}(b)$ inorganic photoresists. 


\section{V.I. Min'ko et al.: Recording of high efficiency diffraction gratings by He-Ne laser}

other conditions for recording the gratings were identical. The relief depths of etched samples of gratings have only 1.1 ratio. It means that the newly developed for $\mathrm{As}_{40} \mathrm{~S}_{20} \mathrm{Se}_{40}$ photoresist etching solution is more selective in comparison to the used for $\mathrm{As}_{40} \mathrm{Se}_{60}$ developer based on the solution of ethylenediamin in dimetilsulphoxide. Besides, $\mathrm{As}_{40} \mathrm{~S}_{20} \mathrm{Se}_{40}$ photoresist photosensitivity is approximately the same as for $\mathrm{As}_{40} \mathrm{Se}_{60}$.

Angular and spectral dependencies of diffractive grating efficiency $\eta$ are the most informative and important from the practical viewpoint. These dependencies for $p$ and $s$-polarization (respectively, parallel and perpendicular arrangement of gratings grooves and electric vector of incident light wave) were measured for studied gratings and are presented in Figs 3 and $4((a)$ - for both figures refers to p-polarization, $(b)-s$-polarization). By comparison of the experimental dependencies with theoretically calculated ones for high-modulated gratings with sinusoidal form of the groove profile $[9,10]$, we can see that maximum values of the diffraction efficiency are comparable to theoretical predictions. Curves for both angular and spectral dependencies, in principal, are in accordance with predicted peculiarities. But for experimental curves, maxima differ from the predicted ones. It indicates that the form of grating profiles is not the ideal sinusoid.
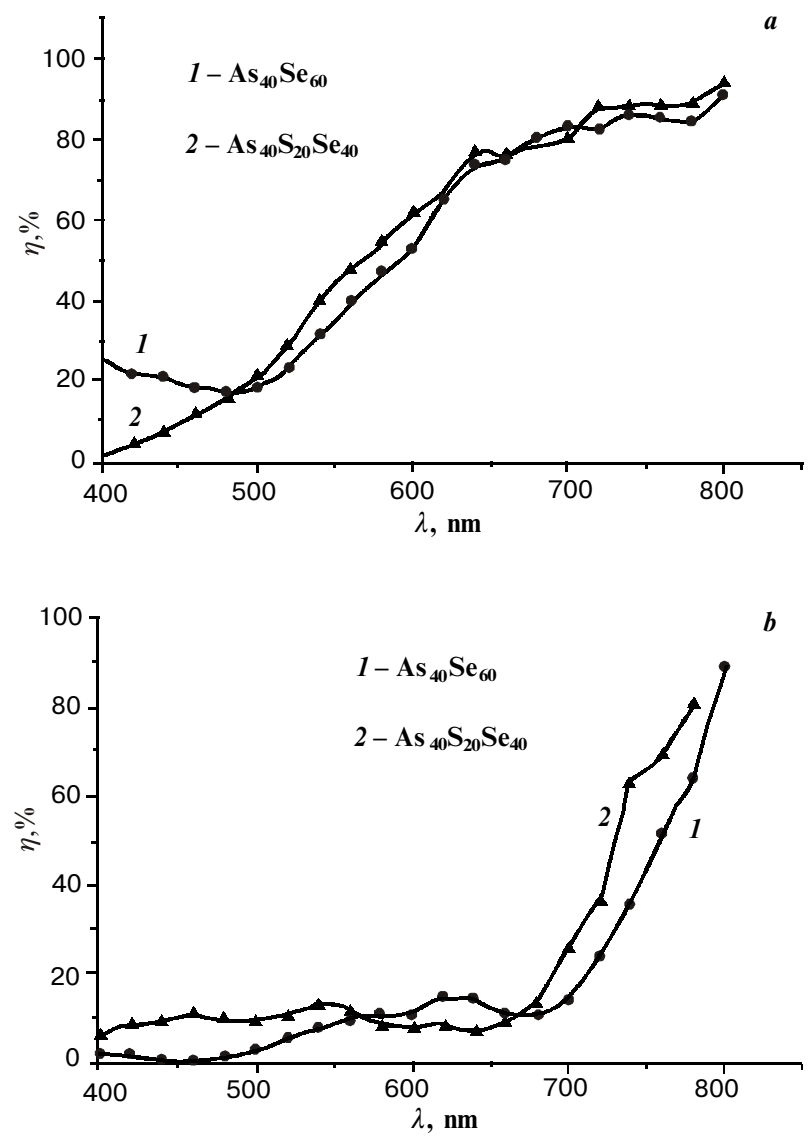

Fig. 3. Spectral dependencies of the diffraction efficiency of the gratings ( $a-p$-polarization, $b-s$-polarization).
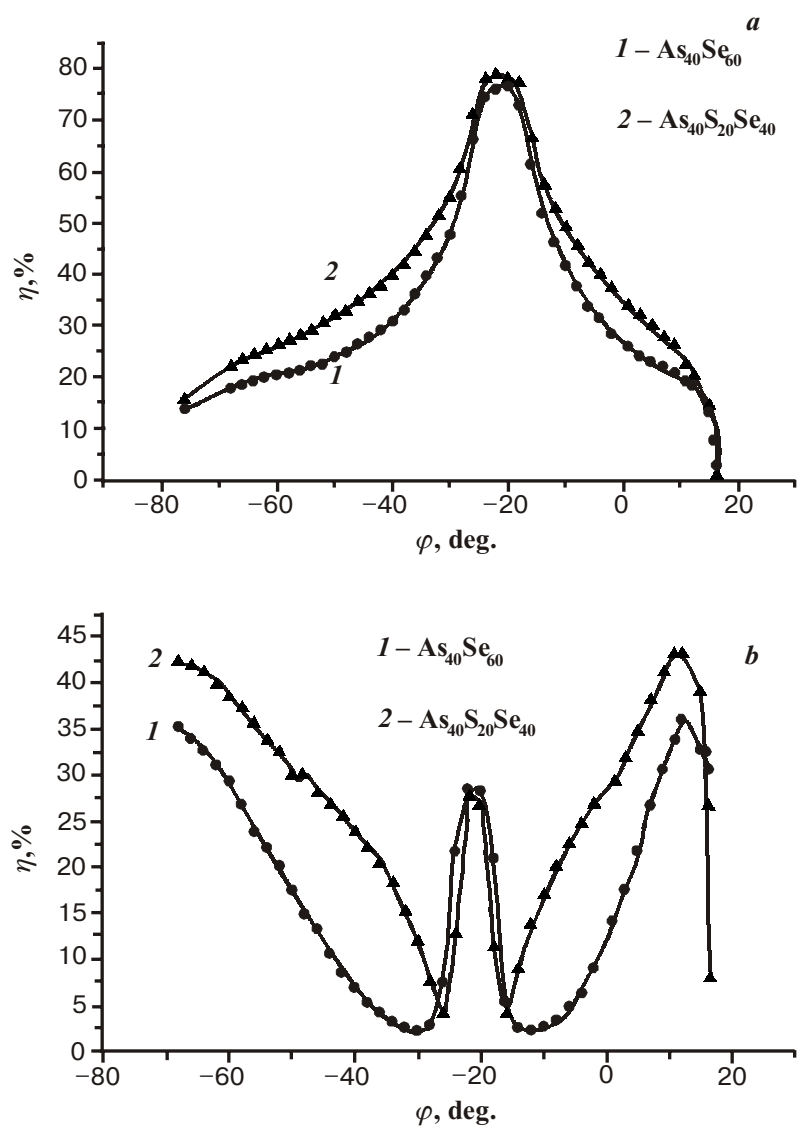

Fig. 4. Angular dependencies of the diffraction efficiency of the gratings ( $a-p$-polarization, $b-s$-polarization).

From the analysis of the data presented in Figs 3 and 4 , one can see that dependencies for grating characteristics recorded using different photoresists are very similar. This is understandable taking into account that the modulation depth and form of grating grooves determine angular and spectral dependencies of diffractive efficiency $[9,10]$.

Thus, we obtained gratings with practically equal parameters on photoresists, optical characteristics of which differ to a great extent. In order to understand this fact, a simulation of the grating production process, using method described in [1] and characteristic curves for the $\mathrm{As}_{40} \mathrm{Se}_{60}$ and $\mathrm{As}_{40} \mathrm{~S}_{20} \mathrm{Se}_{40}$ layers obtained in [3], was carried out. This method enable numerically to compute the form of the groove profile for holographic gratings depending on their production experimental conditions. The light intensity distribution in the photoresist in the course of recording and influence of photoresist optical constants change during exposure on light distribution pattern were taken into account. It is necessary to mention that etching conditions influence the profile shape [1]. In our numerical calculation, varied parameters were the photoresist absorbance at the recorded wavelength, exposure values and time of etching the samples after exposure. Different values of abovementioned param- 


\section{V.I. Min'ko et al.: Recording of high efficiency diffraction gratings by He-Ne laser}

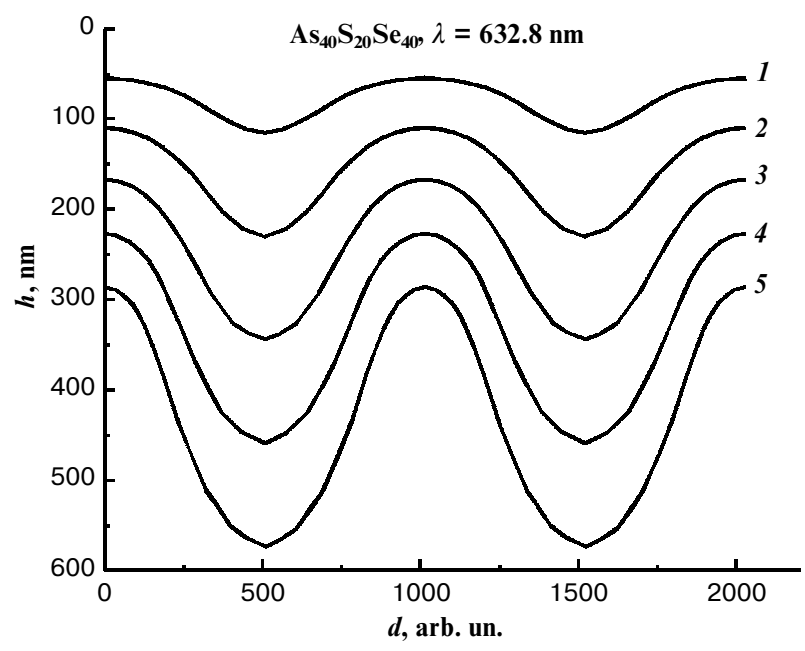

Fig. 5. Dependencies of the relief profile on etching time.

eters, including the experimental ones, were used in calculations and their influence on the profile shape was estimated. The obtained results of numerical modeling has shown that the interference pattern created by recording radiation was reproduced in the photoresist in the region of small photoresist absorption (linear response of the system). Therefore, creation of the sinusoidal relief by strongly absorbed radiation requires narrow combination of optimal parameters affecting conditions of recording. And by the contrast, if the recording is in the region of weakly absorbed radiation, of etching time changing, for example, this leads to changing the relief depth, but not its form. It is clearly seen from Figs 5 and 6 where depicted are the dependencies of groove profile

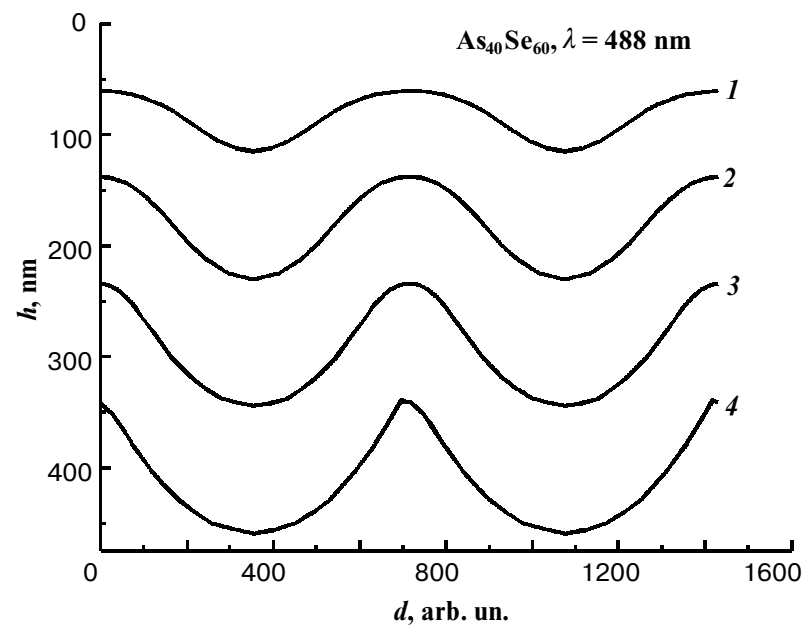

Fig. 6. Dependencies of the relief profile on etching time. shapes on the etching time (curves 1,2,3,4,5 - etching time 2, 4, 6, 8 and $10 \mathrm{~min}$, respectively). (Zero on the vertical axis corresponds to the free interface of photoresist, the initial thickness of photoresist was 600 $\mathrm{nm}$ ). For medium (Fig. 5), absorbance $\alpha$ of which at the recording wavelength is $2.7 \cdot 10^{3} \mathrm{~cm}^{-1}\left(\mathrm{As}_{40} \mathrm{~S}_{20} \mathrm{Se}_{40}, \lambda\right.$ $=632.8 \mathrm{~nm}$ ), the 5 -fold increase in etching time (curve 1 and 5) does not lead to visible changing the relief form, but changes the relief depth. If the photoresist absorbance is increased, the change of the etching time lead to distored relief form. In Fig. 6, results of simulation for photoresist based on $\mathrm{As}_{40} \mathrm{Se}_{60}\left(\alpha=1.24 \cdot 10^{5} \mathrm{~cm}^{-1}\right)$ when recording with the argon laser wavelength $(\lambda=488 \mathrm{~nm})$ takes place. As seen from the figure, the form of groove profile of modulated gratings is changed with the etching time growth.

Taking into consideration dependencies obtained by numerical calculations, we can explain our experimental results. Holographic gratings recording was made by $\mathrm{He}-\mathrm{Ne}$ laser radiation $(\lambda=632.8 \mathrm{~nm})$ on $\mathrm{As}_{40} \mathrm{~S}_{20} \mathrm{Se}_{40}$ and $\mathrm{As}_{40} \mathrm{Se}_{60}$ photoresists, absorbance of which reaches $2.7 \cdot 10^{3} \mathrm{~cm}^{-1}$ and $1 \cdot 10^{4} \mathrm{~cm}^{-1}$, respectively, (region of weak absorbance) and response of media was linear (or close to linear). Both photoresists were exposed using the same light pattern, time of etching was chosen as the time of complete solution of unexposed photoresist layers. In such conditions for both used compositions of photoresists, the gratings with similar $\eta$ values were obtained in accordance with the results of numerical calculations.

\section{Conclusions}

The performed investigations show that the photoresists based on $\mathrm{As}_{40} \mathrm{~S}_{20} \mathrm{Se}_{40}$, in spite of the low absorbance value, are suitable for recording the periodical holographic structures by using helium-neon laser radiation. Use of the newly developed high-selective etching solution enabled to produce high quality reflective diffraction gratings with maximal $\eta$ values close to the calculated ones. The groove profile of diffraction gratings with the maximal $\eta$ values is close to a sinusoid with groove depth about $300 \mathrm{~nm}$. Maximal diffraction efficiency of gratings is $83 \%$ in polarized light and $\sim 75 \%$ in non-polarized one. Numerical computation of grating relief formation enabled us to understand experimental results of grating fabrication using these different photoresist composition.

\section{Acknowledgements}

The authors wish to express their gratitude to Dr A.V. Stronski for valuable discussion and critical reading the manuscript. 


\section{V.I. Min'ko et al.: Recording of high efficiency diffraction gratings by He-Ne laser}

\section{References}

1. I.Z. Indutnyi, A.V. Stronski, S.A. Kostioukevich et al., Holographic optical element fabrication using chalcogenide layers // Optical Engineering, 34(4), pp.1030-1039 (1995).

2. M. Vlcek, P.J.S. Ewen, T. Wagner, High efficiency diffraction gratings in As-S layers//J. of Non-Cryst. Solids, 227230, pp. 743-747 (1998).

3. P.E. Shepeliavyi, S.A. Kostioukevich, I.Z. Indutnyi, A.V. Stronski, Fabrication of periodical structures with the help of chalcogenide inorganic resists // Proc. SPIE, 2291, pp.188192 (1994).

4. I.Z. Indutnyi, S.A. Kostioukevich, V.I. Min'ko et al. Laser lithography utilizing $\mathrm{As}_{2} \mathrm{~S}_{3}$ layers // Optoelektronika Poluprovodnikovaya Tekhnika, 25, pp. $52-59$ (1993), in Russian.

5. P.F. Romanenko, I.I. Robur, A.V. Stronski, I.Z. Indutnyi, The recording of relief-phase holographic gratings utilizing $\mathrm{As}_{2} \mathrm{Se}_{3}$ layers // Ukrainskii Fizicheskii Zhurnal, 36(7), pp. 2028-2031 (1991), in Russian.
6. A.V. Stronski, Photostimulated processes in chalcogenide vitreous semiconductors and their application for the fabrication of the holographic optical elements. Manuscript. Thesis for Dr. Sci. degree, the Institute of Semiconductor Physics, NAS of Ukraine, Kyiv, 2001.

7. P.F. Romanenko, I.I. Robur, A.V. Stronski, Investigation of recording processes of holographic diffractive gratings on $\mathrm{As}_{2} \mathrm{Se}_{3}$ layers by helium-neon laser radiation // Optoelektronika i Poluprovodnikovaya Tekhnika, 27, pp. 47-50 (1994), (in Russian).

8. I.Z. Indutnyi, A.I. Stetsun, Determination of the optical constants of thin absorbing films on a slightly absorbing substrate from photometric measurements // Proc. SPIE, 2113, pp. 5559 (1993).

9. M.C. Hutley, Diffraction gratings, Acad. Press, London, 1982.

10. R.R. Gerke, I.V. Golubenko, T.G. Dubrovina, G.M. Savitsky, Study of reflection properties of hologram diffraction gratings with symmetric profile of lines // Sov. Optika and Spektroskopiya, 58(6), pp. 1318-1322 (1985). 